\title{
Update on the TowerJazz CMOS DMAPS development for the ATLAS ITK
}

\author{
A. Sharma ${ }^{a}$, B. Hiti ${ }^{b}$, C. Solans Sanchez ${ }^{c}$, C.A.M Tobon ${ }^{c}$, C. Riegel ${ }^{d}$, E.J. Schioppa ${ }^{c}$, \\ F. Dachs ${ }^{* c}$, H. Pernegger ${ }^{a}$, I. Asensi Tortajada ${ }^{f g}$, I. Berdalovic $^{c}$, J.W. Van Hoorne ${ }^{h}$, \\ L.S. Argemi ${ }^{i}$, M. Moreno Llacer ${ }^{c}$, N. Wermes ${ }^{j}$, N. Egidos Plaja ${ }^{k}$, P. Riedler ${ }^{c}$, P.

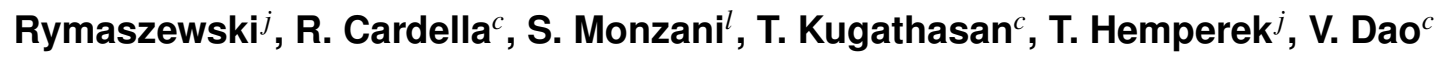 \\ and W. Snoeys ${ }^{c}$ \\ ${ }^{a}$ University of Oxford, ${ }^{b}$ Jozef Stefan Institute, Slovenia, ${ }^{c}$ CERN, ${ }^{d}$ Bergische Universität \\ Wuppertal, ${ }^{e}$ Technische Universität Wien, ${ }^{f}$ University of Valencia, ${ }^{g}$ CSIC, ${ }^{h}$ Austrian \\ Academy of Sciences, ${ }^{i}$ University of Glasgow, ${ }^{j}$ University of Bonn, ${ }^{k}$ University of Barcelona, \\ ${ }^{l}$ INFN
}

The upgrade of the ATLAS tracking detector for the High-Luminosity Large Hadron Collider at CERN requires the development of novel radiation hard silicon sensor technologies. For the development of depleted CMOS sensors for ATLAS we combined small electrodes with minimal capacitance and advanced processing for fully depleted active sensor volume to achieve radiation hard CMOS sensors in line with ATLAS ITk specifications.

Based on initial studies on the prototype sensor "TowerJazz Investigator" we have now developed, produced and tested a first full-size depleted CMOS sensor based on the 180nm TowerJazz imaging process, the so-called "MALTA" sensor. The sensor combines special low-noise/low power front-end using small electrodes with a novel high-speed asynchronous readout architecture to cope with the high hit-rates expected at HL-LHC. The sensor has been produced end of 2017 and initial measurements have been carried out assessing its analog and digital performance. The poster will show efficiency studies performed with the TowerJazz Investigator chip at the CERN SPS test beam facility in 2017 and first measurement results with the new "MALTA" sensor.

The 39th International Conference on High Energy Physics (ICHEP2018)

4-11 July, 2018

Seoul, Korea

${ }^{*}$ Speaker. 


\section{TowerJazz Investigator}

The TowerJazz Investigator chip is a monolithic silicon pixel chip with purely analogue readout and features 134 pixel flavours of varying design[1]. This chip was originally developed to study monolithic CMOS designs for the ALICE experiment at CERN using a modification added to the standard production process which increases radiation hardness while reducing the charge collection time. The chip was then also used to study possible pixel designs for the ATLAS upgrade. Test beam studies show that particular pixel designs maintain high detection efficiency after an irradiation to $10^{15} \mathrm{n}_{\mathrm{eq}} / \mathrm{cm}^{2}$. Specifically, an efficiency of $93.4 \%$ was obtained with a set threshold of $110 \mathrm{e}^{-}$directly applied to analogue wave forms from the pixels (Fig. 1a). Results obtained from these measurements guided the development of a first full-sized digital prototype called MALTA (Monolithic from ALice To Atlas).

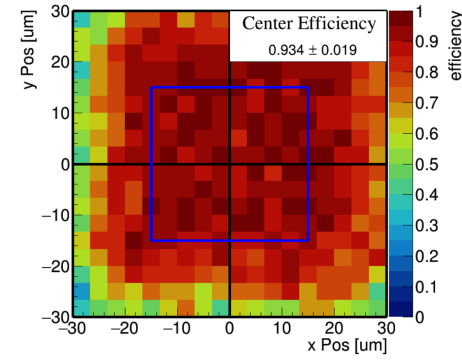

(a) TJ Investigator Efficiency

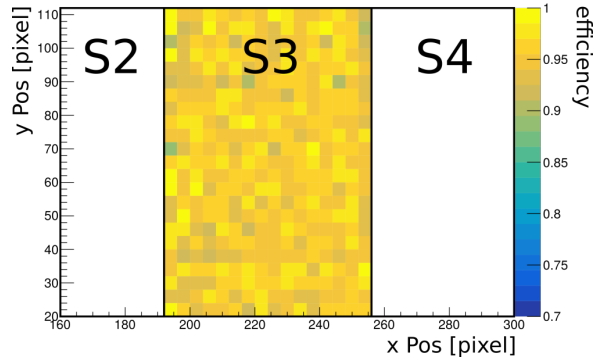

(b) TJ MALTA Efficiency

Figure 1: Test beam measurement results with the TowerJazz Investigator and MALTA chips. Fig.1a: Shown are $2 \times 2$ pixels with $30 \times 30 \mu \mathrm{m}^{2}$ pixel pitch of the Investigator chip. The efficiency is only considered in the central region marked in blue which is not affected by edge effects. Fig.1b: Shown is the global un-irradiated efficiency of the MALTA chip for a specific sector where good performance was observed.

\section{TowerJazz MALTA}

The TowerJazz MALTA chip is the first prototype of a monolithic CMOS pixel chip produced with the $180 \mathrm{~nm}$ process by TowerJazz with a small collection diode design and asynchronous readout[2].

The small collection diode is only $3 \mu \mathrm{m}$ in diameter and this results in a pixel capacitance of $<5 \mathrm{fF}$. This enables very low thresholds of $150 \mathrm{e}^{-}$and an equivalent noise charge (ENC) of $20 \mathrm{e}^{-}$. This in turn allows for a thinner chip which can be fully depleted and is thus faster and more radiation hard. Due to noise issues, current measurements can only be done at considerably higher thresholds. Initial measurements show an un-irradiated global efficiency of $95 \%$ at a threshold of $250 \mathrm{e}^{-}$in a well performing sector of the chip (Fig. 1b).

\section{References}

[1] H. Pernegger et al. First tests of a novel radiation hard CMOS sensor process for Depleted Monolithic Active Pixel Sensors. JINST 12 (2017) P06008

[2] W. Snoeys et al. A process modification for CMOS monolithic active pixel sensors for enhanced depletion, timing performance and radiation tolerance. NIM A 871 (2017) 90-96. 